This item was submitted to Loughborough's Research Repository by the author.

Items in Figshare are protected by copyright, with all rights reserved, unless otherwise indicated.

\title{
Modelling manufacturing processes using Markov chains
}

PLEASE CITE THE PUBLISHED VERSION

https://www.crcpress.com/Safety-and-Reliability-Theory-and-Applications/Cepin-Bris/p/book/9781138629370

\section{PUBLISHER}

(C) CRC Press

\section{VERSION}

AM (Accepted Manuscript)

\section{PUBLISHER STATEMENT}

This work is made available according to the conditions of the Creative Commons Attribution-NonCommercialNoDerivatives 4.0 International (CC BY-NC-ND 4.0) licence. Full details of this licence are available at: https://creativecommons.org/licenses/by-nc-nd/4.0/

\section{LICENCE}

CC BY-NC-ND 4.0

\section{REPOSITORY RECORD}

Leigh, Johanna M., Lisa M. Jackson, Sarah J. Dunnett, Heinz Lugo, Richard Sharpe, Aaron D. Neal, and Andrew A. West. 2017. "Modelling Manufacturing Processes Using Markov Chains". figshare.

https://hdl.handle.net/2134/26018. 


\title{
Modelling Manufacturing Processes using Markov Chains
}

\author{
J.M. Leigh, L. Jackson \& S. Dunnett \\ Department of Aeronautical and Automotive Engineering, Loughborough University, Leicestershire, UK
}

\author{
H. Lugo, R. Sharpe, A. Neal \& A. West
}

The Wolfson School of Mechanical, Electrical and Manufacturing Engineering, Loughborough University, Leicestershire, UK

ABSTRACT: Optimizing manufacturing processes with inaccurate models of the process will lead to unreliable results. This can be true when there is a strong human influence on the manufacturing process and many variable aspects. This study investigates modelling a manufacturing process influenced by human interaction with very variable products being processed. To develop a more accurate process model for such processes radio frequency identification (RFID) tags can be used to track products through the process. The tags record information for each product and this data can be used to produce more accurate models of the manufacturing process. The data produced has been used to create a Markov chain model. This model is used to predict future product paths for use in discrete event simulation. In this case an IT refurbishment company is used as a case study. RFID tags have been utilized to track the IT products moving through the refurbishment process and this information has been used to produce a Markov chain model.

\section{INTRODUCTION}

Modelling manufacturing processes which contain human interactions is difficult and can produce unrealistic views of the process. This is because in many companies the actual manufacturing process that takes place is not as planned when human interaction is involved. Human factors can determine what actually happens, the time it takes and what order it happens in. To produce a more reliable representation of the process more information on what is actually happening is required. This can be found by tracking and recording the process using radiofrequency identification (RFID) tags (Weinstein, 2005). From the data produced from these tags the possible paths which products take in the process can be determined and hence the actual manufacturing process can be defined. Furthermore the data can be used to form Markov chains which can determine what future process routes will look like and the probability of each route. Basing future business simulations on these Markov chains can give a more reliable representation of the business. This reduces the risk of modelling inaccuracies and can help to predict future outcomes and run optimisation more accurately.

The research performed here studies a company which refurbishes IT products. The company has a business model of the manufacturing process which it expects the products to follow. The company has tracked their products through the refurbishment process using RFID tags to determine what processes each product undertakes and to allow each object to be kept track of. The RFID tags communicate the process information of each product to a database software using RFID tag readers. The information from these RFID tags is used in the work presented here to form a Markov chain representation of the business process routes.

Companies' model manufacturing processes for many reasons, including predicting cost (Rehman et al., 1998), predicting resource and material demand and running optimisation studies.

When modelling the data produced from the RFID tags the Markov chain produced gives a large variety of process routes. These are not all true reflections of the routes products take. The data mining process from the RFID tag data is also investigated to allow the development of more precise process models. This process allows thresholds to be set for each route. Hence irregular paths are removed.

The Markov chain is necessary to simulate the product flow. The process is simulated using the Markov chain produced from the data and the results can be compared to the process simulated based on previous perceptions of the business process. The results produced will include the time taken for each product to be processed, the cost of the process and the final destination of products. The Markov chain 
can then be used to test the effects of disallowing transitions, limiting the process paths.

\subsection{Manufacturing}

Manufacturing is the process of using raw materials, components or parts to produce a finished product which meets customers' expectations or requirements (Business Dictionary, 2017). The manufacturing process can be made up of many different activities which are dependent on the industry. The order in which these activities are carried out can be fixed or could be determined by human interaction if it is a highly manual process. The manufacturing process considered here is concerned with the refurbishment of IT products and is a manual process. The company has provided a business flow diagram which it expects products tin reality o follow. Each product enters the process in a different state, hence human interaction is required to determine which activities are required and in what order. It is difficult to build into any model of the process the effects of this human interaction.

Modelling manufacturing process where humans are a factor can lead to inaccurate results. A study by (Baines et al. 2004) looked into improving the inaccuracies in discrete event simulation when considering a highly manual process. This study demonstrated how human performance model could be connected to discrete event simulations.

This research uses the data produced from RFID tags to understand how the manufacturing process changes from the initial business flow model to when implemented on the shop floor. Due to humans implementing the procedure and making decisions on how to proceed.

\subsection{RFID tags}

Automated identification systems are important to allow accurate and timely information on objects on a manufacturing line to be communicated. This supports decision making and ability to control the process (McFarlane et al., 2003). Hence RFID tags are an important development in automated identification systems.

RFID tags are intelligent bar codes. They contain a chip which records data on the product. They are read by an electronic reader. This allows objects to be tracked. They were originally used for tracking cattle but their use has since been expanded. This includes tracking vehicles (Weinstein, 2005), pets (Rieback et al., 2006) and products in the manufacturing process (Sharpe et al., 2014). Concepts for future applications include replacing the till system in grocery shopping with tags and electronic sensors which transmit information to stores and banks (Bonsor \& Felon, 2007), (Långström, 2013). RFID tag technology has been around for decades but only recently has the manufacturing of them been developed to a standard such that the price of the tags enables them to be used on a large scale (Weinstein, 2005).

Previous uses of RFID tags in production lines includes (Sharpe et al., 2014) using RFID tags, along with accelerometers and temperature sensors, in the detection of defects. The aim of this study was to assist in the ability to ensure right first time manufacturing. This produced a leaner manufacturing process.

The research detailed in this paper uses RFID technology to track objects on an IT refurbishment line. The data recorded from the tags includes the product ID, process taking place, start and end time, the type of product being processed and the model. The data is then utilized to determine the process routes and predict future process routes to allow the system to be modelled with a higher accuracy.

\subsection{Markov analysis}

Markov chains and Markov processes are used to model stochastic processes. Markov chains model discrete-time processes and Markov processes models continuous-time processes. They mathematically model a process by showing how the process can move between different stages and the probability of making these transitions. They can be shown in diagrammatic form, figure 1 , or through transition matrices, equation 1. Figure 1 shows a Markov chain model of a process with 2 stages A1 and A2 where the probability of making a transition from stage i to stage $\mathrm{j}$ is given by $\mathrm{q}_{\mathrm{ij}}$.

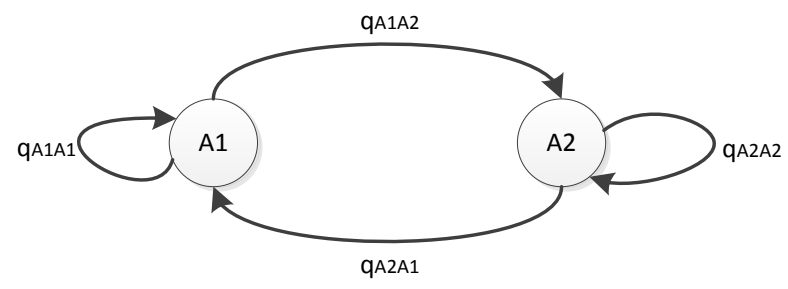

Figure 1. Markov chain diagram.

$$
{ }_{A 2}\left(\begin{array}{cc}
A 1 & A 2 \\
q_{A 1 A 1} & q_{A 1 A 2} \\
q_{A 2 A 1} & q_{A 2 A 2}
\end{array}\right)
$$

The transition matrix in equation 1 shows the probability of transitioning between the row stage to the column stage.

To form a Markov chain model the transition probabilities are required. These are calculated using equation (2). Equation (2) determines the probability of making a transition from stage $i$ to stage $j$, which is represented by $\mathrm{P}_{\mathrm{ij}}$. Where $\mathrm{m}$ is the total number of transitions and $n_{i, j}$ is the number of transitions from $i$ to $j$. 


$$
P_{i, j}=\frac{n_{i, j}}{\sum_{k=1}^{m} n_{i, k}}
$$

\section{MANUFACTURING PROCESS}

\subsection{Overview of process}

The manufacturing process under consideration relates to the end of life phase and is an IT refurbishment process. This contains activities such as visual inspection, functional test and data erasure. The refurbishment process is said to have eight possible stages. These stages are labeled A1, A2, A3, A4, A5, A6, A7 and A8. A1 is assumed by the company to be the starting stage of every product in the refurbishment process as this is the asset tracking process, where each product is logged into the system and given an RFID tag. A2, A3, A4, A5, A6 are intermediary stages. A2 is the visual inspection, A3 is the functional test, A4 is the data erasure, A5 is the cleaning and de-labelling and A6 is the repair. A7 and $\mathrm{A} 8$ are the two possible finishing stages of the process, where $A 7$ is the stripping and scrapping of the product and A8 is the product being sold. The process is designed to be carried out with the stages in the order shown in Figure 2.

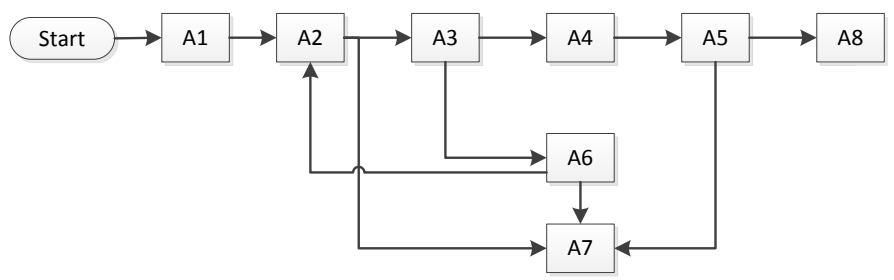

Figure 2. Expected process diagram.

\subsection{Limitations of process}

Following a process review it was clear that the process had high variability and a significant proportion of the time it did not follow the expected process diagram. One reason is due to the variety of IT products being processed leading to different process routes being required depending on the type and state of variability of the products being processed. To optimize the refurbishment process, initial steps are required to better understand the process and elicit the knowledge of the process itself which is currently lacking. To achieve this, the company required more information on the process each product went through to allow more reliable modelling of the system. This was achieved by tracking products using RFID tags. Each IT product was given an RFID tag and each station where the stages are carried out was given an RFID tag reader. The tags were registered on a computer tracking software which was linked to the RFID tag readers. The RFID tags are scanned by the reader as each stage is started and finished and the records of this are transmitted to the computer tracking software. This data can be used to model the process as a Markov chain.

\section{DATA}

\subsection{Finding paths from data}

The data recorded contains the product ID, process, process start and end time, product model and type. There are 99727 data entries in the raw data. An example of the data is given in Table 1 .

Table 1. Data extract from RFID software

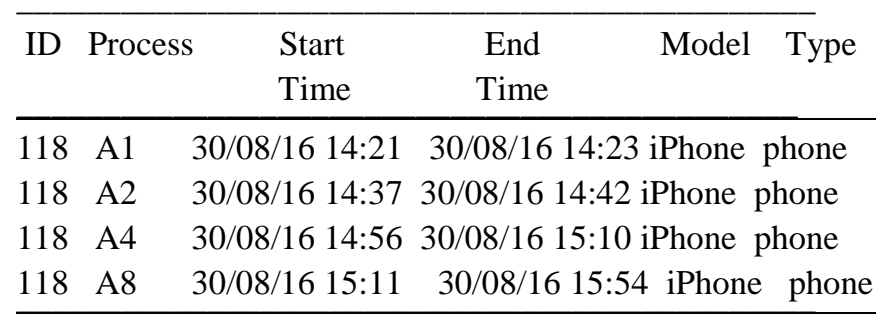

Using this data initial analysis shows the possible stages are $n=\{A 1, A 2, A 3, A 4, A 5, A 6, A 7, A 8\}$. These stages do not show the products leaving the process. Hence an additional stage $\mathrm{AE}$, which is the activity of exiting the process, was added. A product is said to enter this state if there are no more process entries recorded for that product code in the RFID tag data base. The stages are now $\mathrm{n}=\{\mathrm{A} 1, \mathrm{~A} 2, \mathrm{~A} 3$, A4, A5, A6, A7, A8, AE\}. From the data the transitions between each stage were then identified. These are shown in the matrix shown in equation (3). Where each element $n_{i, j}$ shows the number of products which went from stage $i$ to stage $j$. Where $i$ and j range from 1 to 9 corresponding to A1, A2, A3, A4, A5, A6, A7, A8 and AE. Zero shows that no products transferred between stages.

\begin{tabular}{c|ccccccccc} 
& $A 1$ & $A 2$ & $A 4$ & $A 3$ & $A 5$ & $A 6$ & $A 7$ & $A 8$ & $A E$ \\
\hline$A 1$ & 14 & 9940 & 677 & 6530 & 0 & 0 & 0 & 0 & 0 \\
$A 2$ & 0 & 2 & 5839 & 4671 & 490 & 24 & 608 & 5704 & 305 \\
$A 4$ & 0 & 666 & 8 & 649 & 1696 & 1204 & 4 & 6264 & 245 \\
$A 3$ & 0 & 7033 & 4211 & 2 & 1 & 1 & 0 & 965 & 131 \\
$A 5$ & 0 & 0 & 0 & 0 & 0 & 0 & 0 & 2121 & 66 \\
$A 6$ & 0 & 2 & 0 & 429 & 0 & 0 & 793 & 0 & 0 \\
$A 7$ & 0 & 1 & 0 & 0 & 0 & 0 & 0 & 0 & 1404 \\
$A 8$ & 0 & 0 & 1 & 0 & 0 & 58 & 0 & 4832 & 14995 \\
$A E$ & 0 & 0 & 0 & 0 & 0 & 0 & 0 & 0 & 0
\end{tabular}

This information can be used to find the possible transition paths. This is shown in figure 3. 


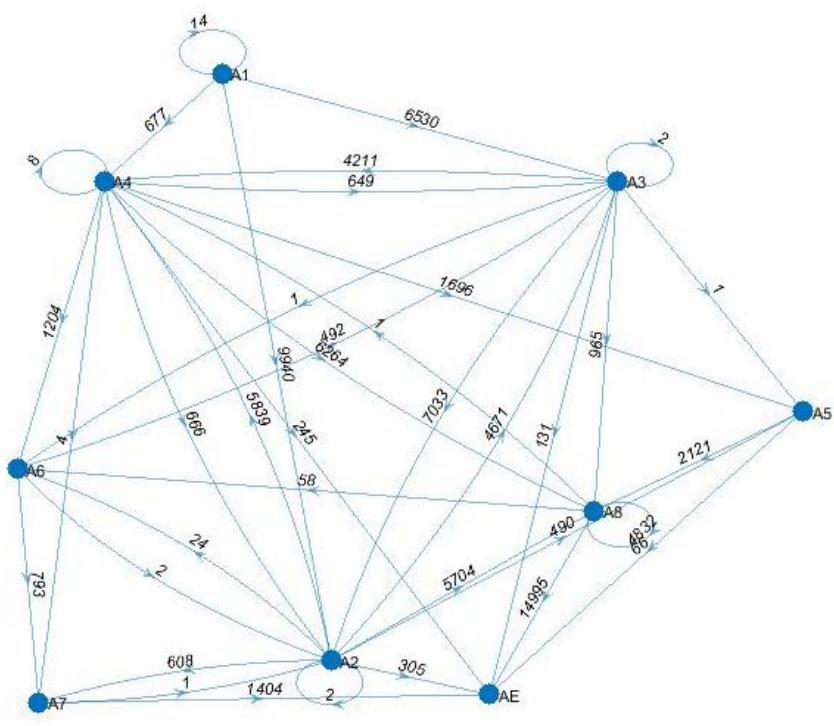

Figure 3. Diagram demonstrating the transitions which occur in the data and the number of times they occur.

This diagram shows the variety of different transitions which can occur. It also shows that some processes are repeated. For example, why are products sold more than once as shown by $\mathrm{n}_{8,8}$. The data identified 4832 transitions between A8 and A8. This could be due to a problem the first time the process was attempted i.e. a sale falls through. The low transition numbers, such as the transition from A2 to A2 and A7 to A2, could be due to anomalies in data.

\subsection{Probabilities}

To represent the process as a Markov chain the probability of transferring between each stage is required. The data shown in equation (3) can be used, alongside equation (2), to determine the probability of making a transition from stage $\mathrm{i}$ to stage $\mathrm{j}, \mathrm{P}_{\mathrm{ij}}$.
Where $\mathrm{m}$ is the total number of transitions, which is equal to 9 in this example.

Calculating the probabilities using equation 3 , for the data under consideration, results in the transition matrix in equation 4 .

\begin{tabular}{c|ccccccccc} 
& $A 1$ & $A 2$ & $A 4$ & $A 3$ & $A 5$ & $A 6$ & $A 7$ & $A 8$ & $A E$ \\
\hline$A 1$ & 0.001 & 0.579 & 0.039 & 0.381 & 0 & 0 & 0 & 0 & 0 \\
$A 2$ & 0 & 0.000 & 0.331 & 0.265 & 0.028 & 0.001 & 0.035 & 0.329 & 0.017 \\
$A 4$ & 0 & 0.062 & 0.001 & 0.061 & 0.158 & 0.112 & 0.000 & 0.597 & 0.023 \\
$A 3$ & 0 & 0.570 & 0.345 & 0.000 & 0.000 & 0.000 & 0 & 0.079 & 0.011 \\
$A 5$ & 0 & 0 & 0 & 0 & 0 & 0 & 0 & 0.970 & 0.030 \\
$A 6$ & 0 & 0.002 & 0 & 0.382 & 0 & 0 & 0.616 & 0 & 0 \\
$A 7$ & 0 & 0 & 0.000 & 0 & 0 & 0 & 0 & 0 & 0.999 \\
$A 8$ & 0 & 0 & 0.000 & 0 & 0 & 0.003 & 0 & 0.988 & 0.754 \\
$A E$ & 0 & 0 & 0 & 0 & 0 & 0 & 0 & 0 & 0
\end{tabular}

Each element of the array, $\mathrm{p}_{\mathrm{ij}}$, shows the probability of making the transition between stage $i$ and stage $j$ to three decimal places. Each row $i$ represents the probability of transitioning from stage $i$ to any of the other stages and hence the sum of each row, except row 9, is unity to the accuracy taken. Row 9 represents the transitions to the other stages from stage $\mathrm{AE}$, which is the stage of exiting the process, and hence all probabilities are zero. This array is represented as a Markov chain diagram. This is shown in figure 4 .

\subsection{Markov chain with thresholds}

The Markov chain shown in figure 4 included all the possible transitions and their probabilities. Some of these transitions occurred at very low probabilities and can be assumed to be anomalies. This could have been caused by scanning the RFID tags incorrectly. These should be removed from the data. This is done by setting a threshold of $0.1 \%$ of transitions.

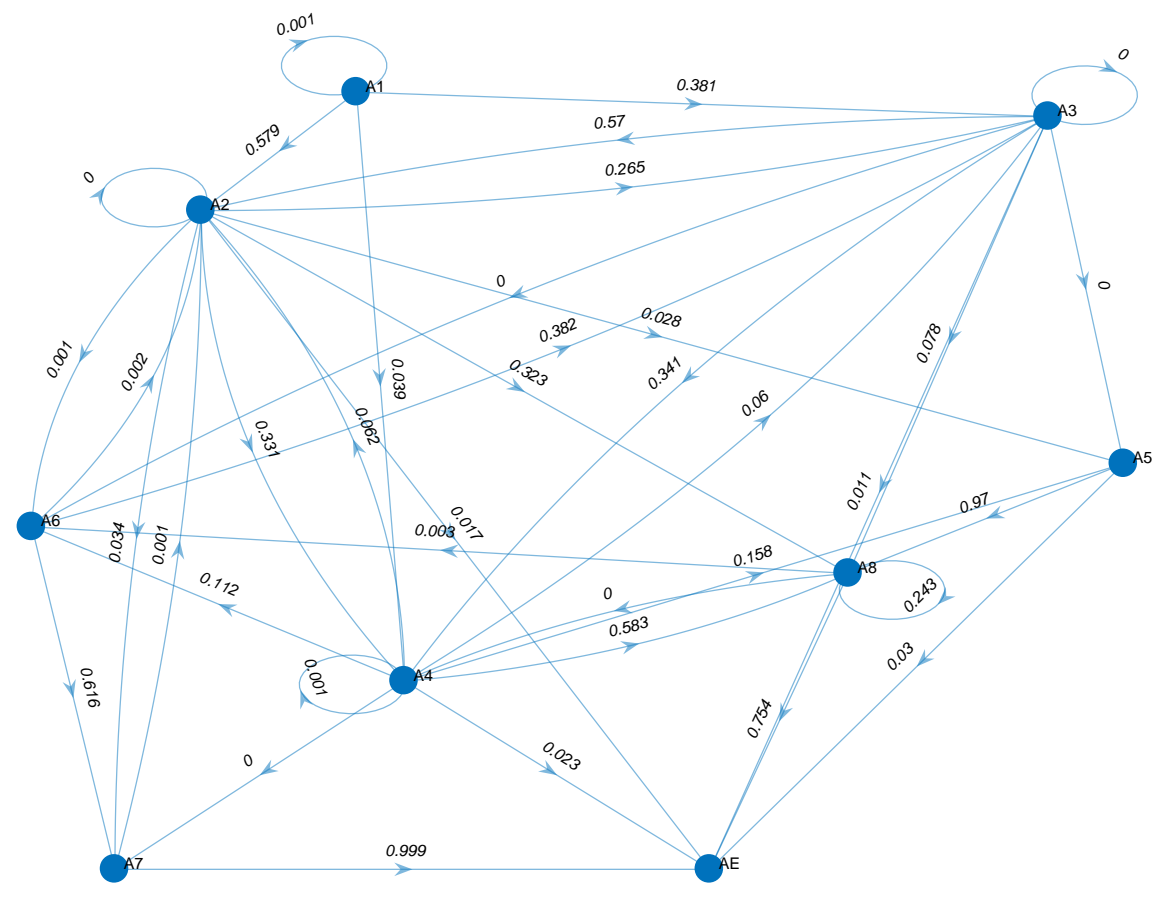

Figure 4. Markov chain using data set showing every transition made in the data. 
This means for the transition to be included in Markov chain there must be at 82 IT products making the same transition. Equation (5) shows the matrix $\left[n_{i j}\right]$ when a threshold of $0.1 \%$ is applied.

\begin{tabular}{c|ccccccccc} 
& $A 1$ & $A 2$ & $A 4$ & $A 3$ & $A 5$ & $A 6$ & $A 7$ & $A 8$ & $A E$ \\
\hline$A 1$ & 0 & 9940 & 677 & 6530 & 0 & 0 & 0 & 0 & 0 \\
$A 2$ & 0 & 0 & 5839 & 4671 & 490 & 0 & 608 & 5704 & 305 \\
$A 4$ & 0 & 666 & 0 & 649 & 1696 & 1204 & 0 & 6264 & 245 \\
$A 3$ & 0 & 7033 & 4211 & 0 & 0 & 0 & 0 & 965 & 131 \\
$A 5$ & 0 & 0 & 0 & 0 & 0 & 0 & 0 & 2121 & 0 \\
$A 6$ & 0 & 0 & 0 & 429 & 0 & 0 & 793 & 0 & 0 \\
$A 7$ & 0 & 0 & 0 & 0 & 0 & 0 & 0 & 0 & 1404 \\
$A 8$ & 0 & 0 & 0 & 0 & 0 & 0 & 0 & 4832 & 14995 \\
$A E$ & 0 & 0 & 0 & 0 & 0 & 0 & 0 & 0 & 0
\end{tabular}

This gives the probability matrix in equation 6 and the Markov chain shown in figure 5.

\begin{tabular}{c|ccccccccc} 
& $A 1$ & $A 2$ & $A 4$ & $A 3$ & $A 5$ & $A 6$ & $A 7$ & $A 8$ & $A E$ \\
\hline$A 1$ & 0 & 0.580 & 0.040 & 0.381 & 0 & 0 & 0 & 0 & 0 \\
$A 2$ & 0 & 0 & 0.331 & 0.265 & 0.028 & 0 & 0.035 & 0.324 & 0.017 \\
$A 4$ & 0 & 0.062 & 0 & 0.061 & 0.158 & 0.112 & 0 & 0.584 & 0.023 \\
$A 3$ & 0 & 0.570 & 0.341 & 0 & 0 & 0 & 0 & 0.078 & 0.011 \\
$A 5$ & 0 & 0 & 0 & 0 & 0 & 0 & 0 & 0.970 & 0.030 \\
$A 6$ & 0 & 0 & 0 & 0.383 & 0 & 0 & 0.617 & 0 & 0 \\
$A 7$ & 0 & 0 & 0 & 0 & 0 & 0 & 0 & 0 & 1 \\
$A 8$ & 0 & 0 & 0 & 0 & 0 & 0 & 0 & 0.244 & 0.756 \\
$A E$ & 0 & 0 & 0 & 0 & 0 & 0 & 0 & 0 & 0
\end{tabular}

This shows that the process taking place is very different from the expected process which is shown in figure 1. For example, from figure 1 stage A2 should definitely follow A1 however figure 5 shows that for only $58 \%$ of the products did stage A2 follow A1 with 38\% going to stage A3 and 4\% going to stage A4. In general the process is much more varied than expected and it is dependent on the product being refurbished.

\section{RESULTS}

The RFID tags produced data which followed what was observed on the shop floor. It allowed the process to be tracked through each stage and recorded useful data to be used in the mathematical modelling of the process. The Markov chain produced in figure 5 shows all the paths taken through the process, starting at A1 and ending at AE, and their likelihood. Overall there are 110 possible paths. The shortest consists of three activities and the longest is nine activities. The most likely path is $s=\{\mathrm{A} 1, \mathrm{~A} 2$, A8, AE $\}$ which involves Asset track, visual inspection, sold and then exits the system. This path has the probability of 0.1417 . The second most likely path is $s=\{A 1, A 2, A 4, A 8, A E\}$ which involves Asset track, visual inspection, data erasure, sold and then exits the system. This path has the probability of 0.0845 of occurring.

Given the 110 possible paths there are many more product routes than assumed by the company. Using this Markov diagram will allow more accurate simulation of the process.

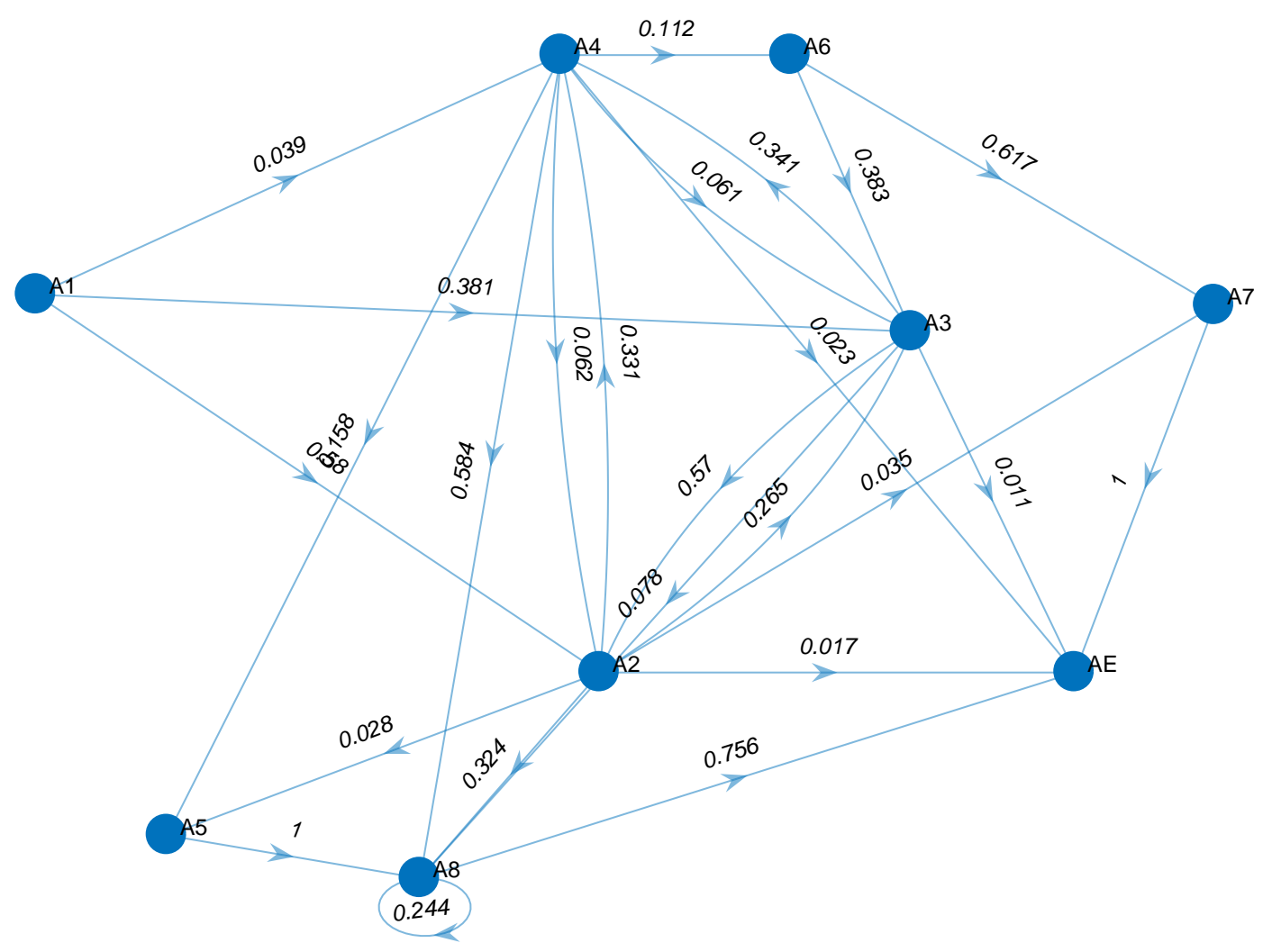

Figure 5. Markov chain using threshold. 


\section{CONCLUSION}

Using RFID tags to track the manufacturing process and using this information to create a Markov chain diagram can lead to a more accurate representation of the system process. Using theMakov model to simulate the process route each product takes will lead to more reliable and accurate results. This in turn can lead to better optimization of the process.

To create the Markov chain diagram from the RFID data it is necessary to consider only transitions which occur more than a threshold value. Considering all transition will lead the simulation of paths which are not possible with a low probability.

Future investigation could highlight the differences in the product paths for different product types and for the different products themselves, ensuring that the product process is correct. This will allow investigation into the effect of changing product lines on the process time. Another line of investigation includes determining the type of processes which RFID tags can track, as some processes may damage the tags.

\section{ACKNOWLEDGEMENTS}

The cooperation of the IT refurbishment company which supported the data collection of this project is gratefully acknowledged along with Richard Sharpe and Aaron Neal who developed the software to collect the data. The authors would also like to thank Engineering and Physical Sciences Research Council for the grant [EP/K014137/1].

\section{REFERENCES}

Baines, T., Mason, S., Siebers, PO., Ladbrook, J., 2004. Humans: the missing link in manufacturing simulation?. Simulation Modelling Practice and Theory. 12(7-8): 515-536.

Bonsor K. \& Fenlon W. 2007. "How RFID Works" 5 November $2007 . \quad$ HowStuffWorks.com. <http://electronics.howstuffworks.com/gadgets/high-techgadgets/rfid.htm> 14 December 2016.

BusinessDictionary.com. Manufacturing. Retrieved January 03, 2017, from BusinessDictionary.com website: http://www.businessdictionary.com/definition/manufacturin g.html

Långström, H. 2013. Grocery industry operations are facing a real paradigm shift. RFID ARENA, accessed 12/2016.

McFarlane, D., Sarma, S., Chirn, J.L., Wong, C. and Ashton, K. 2003. Auto ID systems and intelligent manufacturing control. Engineering Applications of Artificial Intelligence, 16(4), pp.365-376.

Rieback, M.R., Crispo, B. and Tanenbaum, A.S. 2006. The evolution of RFID security. IEEE Pervasive Computing, 5(1), pp.62-69.

Rehman, S. and Guenov, M.D., 1998. A methodology for modelling manufacturing costs at conceptual design. Computers \& Industrial Engineering, 35(3), pp.623-626.

Sharpe, R., Banwell, G., Conway, P., West, A. 2014. Sensorenabled PCBs to aid right first time manufacture through defect prediction. Presented at the 16th Electronics Packaging Technology Conference, (EPTC 2014), Singapore, 35th Dec., pp. 366-371.

Weinstein, R. 2005. RFID: a technical overview and its application to the enterprise. IT professional, 7(3), pp.27-33. 